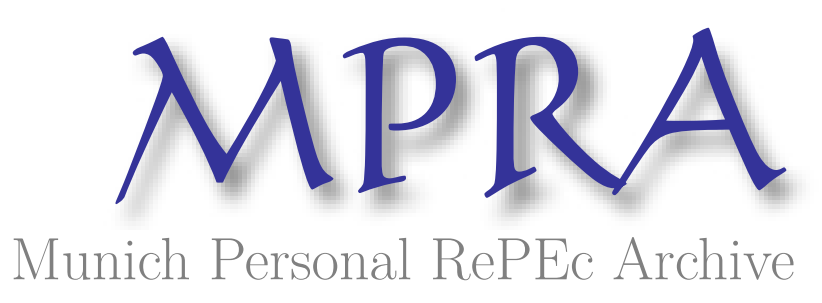

\title{
Survey on Child Labour Statistics
}

Reggiani, Tommaso

Università di Milano-Bicocca - Department of Economics

4 June 2008

Online at https://mpra.ub.uni-muenchen.de/9148/

MPRA Paper No. 9148, posted 16 Jun 2008 06:27 UTC 


\title{
METODOLOGIE STATISTICHE
}

\section{PER LA RiLeVAZIONE DEL LAVORO MinORILE}

\author{
Tommaso Reggiani * \\ (DRAFT - 04/06/2008)
}

\begin{abstract}
(ENG) SURVEY ON CHILD LABOUR STATISTICS.

This paper aims to provide a review on key methodological issues regarding two major international statistical approaches, which characterize the statistics on Child Labour, especially in poor countries. In the first section, we summarises and analyse some key concepts about the international definition on Child Labour. In the second section we analyze the methodology "Statistical Information and Monitoring Programme on Child Labour" (SIMPOC) by International Labour Organization (ILO). In the third section, we describe an alternative methodology promoted by World Bank (WB) identified through the "Living Standard Measurement Survey" (LSMS). In conclusion, in the fourth section, we briefly describe the Italian experience, reporting the major methodological implications emerged during the experimentation of the statistical research project "Children and Work" carried out in Italy by ISTAT (Italian National Institute of Statistics).
\end{abstract}

(ITA) Metodologie Statistiche PER LA RiLEVAZIOnE DEl LAVORO MinORILE. Il presente lavoro si pone l'obiettivo di fornire una rassegna in chiave metodologica riguardante i due maggiori approcci statistici internazionali, che caratterizzano le rilevazioni statistiche circa le dinamiche di lavoro minorile, in particolar modo presso i paesi in via di sviluppo. Nella prima sezione del lavoro, analizzeremo in modo sintetico alcuni riferimenti miliari circa la definizione internazionale di lavoro minorile. Nella seconda sezione, analizzeremo la metodologia "Statistical Information and Monitoring Programme on Child Labour" (SIMPOC) promossa da International Labour Organization (ILO). Essa si presenta attualmente come la migliore metodologia statistica disponibile, circa la stima delle dinamiche di lavoro minorile a livello globale. Nella terza sezione, descriveremo invece la principale metodologia alternativa, promossa da World Bank (WB) ed individuata tramite la "Living Standard Measurement Survey" (LSMS), tesa a stimare il rischio dei minori, di cadere nella condizione di lavoro minorile. In conclusione, all'interno della quarta sezione, descriveremo brevemente l'esperienza italiana, con l'obiettivo di presentare le maggiori implicazioni metodologiche emerse durante l'elaborazione della ricerca statistica "Bambini e Lavoro" (2002) effettuata da ISTAT.

\footnotetext{
* Specializzando in Economia Politica, Università degli Studi di Milano-Bicocca. e-mail: <tommaso.reggiani@gmail.com>.

Il presente paper è stato presentato all'interno del corso "Modelli Statistici per il Mercato del Lavoro", Prof.ssa Mariangela Zenga, UMB a.a. 2007/2008.

Si ringraziano Mariangela Zenga, Beatrice Petrovich e Stefano Ramelli, per i preziosi commenti.
} 
"Tre cose ci sono rimaste del paradiso:

le stelle, i fiori e i bambini"

Dante Alighieri

\section{Definizione Internazionale di Lavoro Minorile}

Il lavoro minorile è un fenomeno complesso. Non tutti i lavori svolti dai bambini possono essere classificati come lavoro minorile ${ }^{1}$. Occorre operare una distinzione tra lavoro minorile propriamente inteso ed altre attività affini, tuttavia considerabili quale parte di un naturale processo di socializzazione ed interazione con la comunità di riferimento.

Bambini lavoratori sono coloro dunque (i) che entrano effettivamente nel mercato del lavoro formale in età precoce, oppure (ii) svolgono un carico troppo gravoso - in relazione alla loro età - di incombenze famigliari.

Anche i maggiori dispositivi giuridici sviluppati sino ad oggi, si orientano nella direzione di concepire il lavoro minorile in questa doppia accezione.

Un rapido sguardo riguardante l'iter e l'evoluzione storica degli strumenti giuridici internazionali - che sino ad oggi hanno contribuito a costituire la base per le relative legislazioni nazionali in materia di lavoro minorile - ci consente di verificare le differenti sfumature concettuali che hanno caratterizzato lo sviluppo della definizione di lavoro minorile.

Il primo tentativo di formalizzare il concetto di lavoro minorile presso una convenzione internazionale, è stato promosso da International Labour Organization (ILO), già durante il proprio congresso di fondazione risalente al 1919.

A partire da questa prima formalizzazione internazionale, che poneva a quattordici anni l'età minima ai fini dell'impiego lavorativo integrale (sia nel settore pubblico che nel settore privato), nel 1973 si perviene alla stesura della Convenzione ILO n.138: in questo rinnovato (ma non molto innovativo) contesto, il concetto di

\footnotetext{
${ }^{1}$ Approfondita disamina in Buffa, Caracuta (2005).
} 
lavoro minorile, si basa ancora esclusivamente sulla legittimità o meno di poter operare presso il mercato del lavoro formalmente inteso.

Un approccio più globale e completo, è stata promosso attraverso la stesura del Codice sui Diritti dell'Infanzia (Rights of the Child - CRC) in sede UN (United Nations) nel 1979.

Il più ampio approccio CRC/UN del 1979, è stato successivamente adottato da ILO nel 1999 attraverso la Convenzione ILO n.182, focalizzata circa le peggiori forme di lavoro minorile ${ }^{2}$.

Queste tre analisi riguardanti il lavoro minorile, rispettivamente, espresse in Convenzione ILO n.138 (1973), Codice sui Diritti dell'Infanzia CRC/UN (1979) ed in particolare presso la più recente Convenzione ILO n.182 (1999), costituiscono l'attuale base ai fini della definizione internazionale del concetto di lavoro minorile.

Internazionale Figura 1: Definizione di Lavoro Minorile (L'area scura individua lo status di lavoro minorile, l'area chiara individua attività para-lavorative di intensità accettabili)

\begin{tabular}{|c|c|c|c|c|}
\hline Up to age 18 & $\begin{array}{l}\text { Dangerous or haz- } \\
\text { ardous work } \\
\text { Worst forms of } \\
\text { child labour }\end{array}$ & Frall-time work & $\begin{array}{l}\text { Part time work } \\
\text { in laborr mar- } \\
\text { ket and voca- } \\
\text { tional training }\end{array}$ & $\begin{array}{l}\text { Household } \\
\text { work if not } \\
\text { interfering } \\
\text { with school or }\end{array}$ \\
\hline $\begin{array}{l}\text { Up to age } 14 \\
\text { or } 15 \text { or age of } \\
\text { completed } \\
\text { compulsory } \\
\text { education (if } \\
\text { higher) }\end{array}$ & & $\begin{array}{l}\text { Full-time work } \\
\text { in labous max- } \\
\text { ket }\end{array}$ & & health \\
\hline $\begin{array}{l}\text { Up to age } 12 \\
\text { of } 13\end{array}$ & & & $\begin{array}{l}\text { Any work in } \\
\text { labons market }\end{array}$ & \\
\hline
\end{tabular}

\footnotetext{
${ }^{2}$ ILO n.1982 art. $3-«[\ldots]$ l'espressione "forme peggiori di lavoro minorile" include : tutte le forme di schiavitù o pratiche analoghe alla schiavitù, quali la vendita o la tratta di minori, la servitù per debiti $\mathrm{e}$ l'asservimento, il lavoro forzato o obbligatorio, compreso il reclutamento forzato o obbligatorio di minori ai fini di un loro impiego nei conflitti armati; l'impiego, l'ingaggio o l'offerta del minore a fini di prostituzione, di produzione di materiale pornografico o di spettacoli pornografici; l'impiego, l'ingaggio o l'offerta del minore ai fini di attività illecite, quali, in particolare, quelle per la produzione e per il traffico di stupefacenti, così come sono definiti dai trattati internazionali pertinenti; qualsiasi altro tipo di lavoro che, per sua natura o per le circostanze in cui viene svolto, rischi di compromettere la salute, la sicurezza o la moralità del minore».
} 


\section{International Labour Organization - SIMPOC "Statistical Information and Monitoring Programme on Child Labour"}

\subsection{Introduzione}

International Labour Organization (ILO - Organizzazione Internazionale del Lavoro), attraverso il proprio programma International Programme on the Elimination of Child Labour (IPEC - Programma Internazionale Eliminazione del Lavoro Minorile), in questo ultimo decennio, ha contribuito in modo fondamentale al fine di trasformare la lotta contro il lavoro minorile in una tematica di domino pubblico a livello globale ${ }^{3}$.

Il gruppo di ricerca IPEC - costituitosi in seno a ILO a partire del 1997 - sta attualmente lavorando con più di 80 stati, al fine di prevenire ed eliminare una piaga che ancor oggi colpisce milioni di bambini e minori in tutto il mondo.

Anche a causa della consistente espansione delle differenti attività condotte da IPEC, è progressivamente sorta l'esigenza di poter disporre di dati ed informazioni sempre più dettagliate ed idonee, al fine di essere impiegate presso l'elaborazione di rigorose indagini a carattere statistico ${ }^{4}$.

Il miglioramento delle tecniche statistiche, inerenti l'osservazione e l'analisi del fenomeno riguardante il lavoro minorile a livello mondiale, è stata individuata da ILO quale chiave principale atta alla definizione ed allo sviluppo di efficaci ed efficienti interventi di policy, finalizzati al contrasto di questo fenomeno, avente gravi risvolti sia a livello umano che sociologico ed economico.

A partire dal 1998, le informazioni statistiche riguardanti il programma di monitoraggio Statistical Information and Monitoring Programme on Child Labour (SIMPOC) condotti ILO/IPEC, hanno consentito la produzione di dati affidabili, completi e comparabili a livello internazionale ${ }^{5}$. Contemporaneamente, grazie al prezioso ruolo di coordinamento scientifico a cura di IPEC presso i differenti istituti statistici nazionali, si è incrementata notevolmente la capacità di ogni singolo paese di raccogliere, elaborare, analizzare e diffondere dati statistici circa il fenomeno del lavoro minorile anche a livello micro e locale. In questi dieci anni di attività, il programma di monitoraggio statistico SIMPOC ha analizzato più di 80 paesi (principalmente PVS) che si distinguono per l'alto tasso di lavoro minorile.

\footnotetext{
${ }^{3}$ ILO (2002).

${ }^{4}$ ILO (2007a).

${ }^{5}$ ILO (2006b).
} 
Figura 2: schema ILO "Theme on Child Labour"

\begin{tabular}{|c|}
\hline \multicolumn{1}{|c|}{ ILO } \\
$\begin{array}{c}\text { International Labour Organization } \\
\text { (agenzia internazionale - 1919) }\end{array}$ \\
\begin{tabular}{|c|} 
IPEC \\
International Programme on the Elimination of Child Labour \\
(progetto - 1997)
\end{tabular} \\
Statistical Information and Monitoring Programme on Child Labour \\
(metodologia statistica - 1998)
\end{tabular}

\subsection{Metodologia statistica}

La corretta tecnica di raccolta ed analisi dei dati generati attraverso l'indagine SIMPOC $^{6}$, costituisce sicuramente l'aspetto più critico di questo progetto.

L'obiettivo principale dell'indagine SIMPOC, è quello di rendere disponibili idonei indicatori statistici, circa il lavoro minorile, atti rispettivamente a:

i) Misurare l'incidenza e le dimensioni del lavoro minorile presso una determinata nazione.

ii) Dar conto delle cause che determinano l'emergere di tale fenomeno presso le differenti realtà nazionali.

iii) Stimare l'efficacia di eventuali programmi di intervento e/o policy, atte a contrastare tale fenomeno.

Al fine di condurre ed applicare in modo rigoroso la metodologia SIMPOC, è stato formalizzato un apposito questionario ${ }^{7}$, composto da serie di batterie di domande fondamentali, tale da poter generare benefiche sinergie se reso complementare alle tradizionali indagini riguardante le forze lavoro, presso le famiglie caratterizzate dalla presenza di minori.

Gli obiettivi generali della metodologia SIMPOC comprendono quindi lo sviluppo di indicatori standard, circa le dinamiche di lavoro minorile a livello delle singole nazioni, nonché la misura e la quantificazione dell'incidenza di tale fenomeno,

\footnotetext{
${ }^{6}$ ILO (2004a), ILO (2004b).

${ }^{7}$ ILO (2007b) - allegato.
} 
al fine di stimare l'impatto e gli effetti che potrebbero esprimere i diversi programmi e policy di intervento.

Sin dalla sua istituzione, SIMPOC è stato comunemente accettato quale punto di riferimento a livello globale, circa lo sviluppo metodologico di strumenti statistici atti a dar conto degli effetti determinati dal fenomeno di lavoro minorile.

Una delle principali svolte metodologiche proposte da SIMPOC, consta nell'ampio impiego di variabili proxy, al fine di stimare l'entità del fenomeno, minimizzando così il rischio di incorrere in dannosi bias o distorsioni, causate da un ridondante impiego di interviste rivolte direttamente ai minori ${ }^{8}$.

Importanti sviluppi hanno avuto luogo anche presso le procedure di campionamento 9 : il protocollo SIMPOC prevede modalità di campionamento stratificato a tre stadi, al fine di assicurare un numero sufficientemente significativo di osservazioni, sia di bambini lavoratori, che di bambini regolarmente impegnati in attività scolastiche. Le unità di primo stadio (ups) sono costituite dalle aggregazioni amministrative di ampio raggio (es. regioni) ${ }^{10}$, le unità di secondo stadio (uss) sono costituite dalle unità amministrative a rilevanza locale (es. comuni/villaggi), infine le unità di terzo stadio (uts) sono costituite dai singoli nuclei famigliari. A seconda dello scenario nazionale di riferimento, si prevede l'eventuale definizioni di unità autorappresentative.

Inoltre, al fine di dar conto in via preliminare di alcune caratteristiche salienti circa il lavoro minorile a livello prettamente locale, SIMPOC prevede l'effettuazione di indagini non sistematiche (Rapid Assessment Methodology - RA) condotte in collaborazione con $\mathrm{UNICEF}^{11}$.

Durante le rilevazioni preliminari (Rapid Assessment Methodology - RA) presso le famiglie - effettuate anch'esse tramite appositi questionari - vengono raccolti dati inerenti una vasta serie di caratteri socio-economici e demografici, finalizzati a permettere la corretta stratificazione del campione sul quale si baserà l'indagine SIMPOC vera e propria.

La dimensione campionaria prevista dalla metodologia SIMPOC è relativamente estesa, fino a arrivare a coprire 10.000 famiglie. Il protocollo SIMPOC prevede una

\footnotetext{
${ }^{8}$ ILO (2004b).

${ }^{9}$ Verma (forthcoming).

${ }^{10}$ Si applica il criterio PPS (Probability Proportional to Size), Fabbris (1989).

${ }^{11}$ ILO, UNICEF, WB (2001), ILO (2006a).
} 
serie di dettagliati e scrupolosi standard, atti alla corretta compilazione dell'elenco completo delle famiglie che entreranno a far parte del campione, al fine di consentire una corretta rappresentazione di tutte le differenti fasce socio-economiche che compongono e caratterizzano la nazione oggetto di studio.

Il questionario SIMPOC $^{12}$, sottoposto alle famiglie selezionate presso il campione finale, è composto da una molteplicità di batterie di domande, suddivise in sezioni autonome.

La prima parte del questionario è rivolta prettamente al capo famiglia, ed è predisposta al fine di ottenere informazioni circa i caratteri demografici ed economicofinanziari che caratterizzano il nucleo famigliare.

La seconda sezione, destinata prettamente alle madri, riguarda le attività svolte e le condizioni di salute che riguardano i figli in età pre-scolare.

Una terza sezione ad-hoc, è di norma riservata direttamente ad ogni minore del nucleo famigliare in età compresa fra i 6 ed i 17 anni, tesa a raccogliere dati ed elementi qualitativi circa la loro situazione scolastica piuttosto che lavorativa.

Lo standard SIMPOC utilizza l'approccio atto a determinare se un soggetto (minore) è stato economicamente/produttivamente attivo nel corso degli ultimi dodici mesi. A livello internazionale, questo orizzonte temporale è normalmente impiegato nell'attività di mappatura della dinamica salariale, dell'occupazione e della disoccupazione specificatamente presso il comparto agricolo. Questa precisa scelta metodologica trova forte fondamento, dato il carattere prettamente agricolo che caratterizza le economie dei PVS.

Un ulteriore aspetto caratterizzante l'approccio SIMPOC, consta nella propensione a sviluppare questionari per quanto più possibile standardizzati ed omogenei, tali da poter essere efficacemente integrati presso le tradizionali rilevazioni riguardanti le forze di lavoro a livello nazionale. In considerazione del fatto che la metodologia SIMPOC è implementata principalmente presso i PVS - tipicamente caratterizzati da uno scarso livello tecnologico - la compilazione del questionario è affidata a rilevatori direttamente e personalmente impegnati sul campo (nella maggior parte dei casi, si esclude l'adozione di metodologia CATI e/o CAPI).

Lo sviluppo circa la tecnica statistica atta alla stima, di natura qualitativa, riguardante le peggiori condizioni di lavoro dei bambini, costituisce una sfida

\footnotetext{
${ }^{12}$ ILO (2007b) - allegato.
} 
metodologica di primo spessore. Infatti, la stima relativa a questa particolare dimensione, non è direttamente ottenibile attraverso le tradizionali indagini basate sui nuclei famigliari. Tuttavia è parzialmente possibile ovviare a questa carenza, attraverso la somministrazione di appositi questionari ${ }^{13}$ direttamente ai referenti delle differenti attività produttive che si distinguono per un alto impiego di lavoro minorile. Il vantaggio di questa metodologia consta nella particolare attitudine nello descrivere con successo, i settori produttivi ed i comparti economici, presso i quali si addensa maggiormente la dinamica di lavoro minorile.

Per quanto riguarda la stima del tasso di pericolosità che caratterizzano le mansioni ricoperte da minori, le indagini basate sui dati emersi dai questionari sottoposti ai nuclei famigliari, risultato ampiamente inadeguati: anche in questo caso, un'analisi diretta del mercato del lavoro e dei suoi principali operatori, è grado di fornire dati indicativi ed attendibili a questo stretto proposito.

\subsection{L'Evoluzione}

Il presente paragrafo si prefigge di analizzare l'evoluzione di SIMPOC, delineandone conseguentemente alcune prospettive che lo caratterizzeranno in futuro.

In primis, descriveremo le fasi ed i risultati intermedi che hanno caratterizzato l'affermarsi di questo innovativo progetto statistico.

Infine, tratteggeremo alcune delle maggiori prospettive che accompagnano SIMPOC verso la " $18^{\text {th }}$ International Conference of Labour Statisticians" (ICLS) del novembre-dicembre 2008: in questa sede, infatti, si definiranno le priorità future che caratterizzeranno l'approccio SIMPOC nel prossimo decennio.

In questi dieci anni di attività, SIMPOC ha raccolto, elaborato ed analizzato dati pertinenti il lavoro minorile specialmente presso i PVS.

Volendo schematizzare, possiamo distinguere quattro fasi principali di sviluppo $^{14}$ che hanno caratterizzato SIMPOC: (i) lo start-up, (ii) una successiva rapida espansione accompagnata dalla diversificazione delle prospettive d'indagine, (iii) il consolidamento delle metodologie statistiche (iv) la definizione - ancora in itinere - di un protocollo statistico standardizzato a livello globale riguardante la rilevazione del lavoro minorile in tutte le sue differenti forme e varianti.

\footnotetext{
${ }^{13}$ Anch'essi caratterizzati da un ampio ricorso a variabili proxy/strumentali.

${ }^{14}$ ILO (2007a).
} 
Nonostante le fasi di sviluppo sono sempre state molto graduali e sfumate, tuttavia è possibile porre in evidenza alcune caratteristiche salienti.

\section{(i) 1998 - 2000: start-up}

Il protocollo statistico SIMPOC è varato da ILO/IPEC nel febbraio 1998 grazie allo stanziamento di fondi istituzionali da parte delle grandi agenzie mondiali: ILO, UNDP, WB, IMF. L'iniziativa si propone come risposta concreta ed operativa nei confronti della formale richiesta di dati affidabili circa il lavoro minorile, avanzata dalla comunità scientifica internazionale - impegnata principalmente nell'ambito statistico/economico - , emersa presso UN Conference on Child Labour ${ }^{15}$, tenutasi a Oslo nel 1997.

Nella sua fase di avvio, SIMPOC beneficia della proficua collaborazione fra accademici di livello internazionale e tecnici afferenti all' ILO Bureau of Statistics (STAT).

In sintesi, l'architrave del progetto statistico - in questa fase - è caratterizzato dei seguenti obiettivi:

i) Raccolta, analisi e diffusione di dati ufficiali, circa le dinamiche di lavoro minorile.

ii) Creazione di una base di dati, articolata su scala mondiale.

iii) Definizione, sia a livello nazionale che a livello globale, di prassi operative e metodologie statistiche, ampiamente condivise ed accettate.

iv) Sviluppo di indicatori standardizzati e sintetici, circa il lavoro minorile.

(ii) 2001-2003: fase di rapida espansione e diversificazione dell'indagine

IPEC e SIMPOC intensificano notevolmente la propria attività statistica, tuttavia si riscontravano alcune carenze, sia di carattere tecnico, che di carattere amministrativo causate dal sottodimensionamento della struttura organizzativa. Al fine di perseguire standard di efficienza, ILO finanzia l'ampliamento dell'organico dedito al progetto. Questo nuovo sforzo finanziario consente la costituzione di rappresentanze ed uffici SIMPOC decentrati, dedicati allo studio ed al monitoraggio delle realtà regionali più critiche da punto di vista del lavoro minorile: Africa, Asia, America Latina.

\footnotetext{
${ }^{15}$ UN Conference on Child Labour in Oslo 1997, web-page:

< http://www.un.org/News/Press/docs/1997/19971031.GASH3430.html >.
} 
Nel 2003, viene avviato il collegio di valutazione SIMPOC External Advisory Committee (SEAC), composto da quindici esperti a livello mondiale in ambito statistico.

A loro è affidato il fondamentale incarico di rendicontare circa l'efficienza e l'efficacia del sistema statistico SIMPOC.

L'autorevole lavoro svolto da questo nuovo nucleo valutativo, permetterà a SIMPOC di instaurare proficue collaborazioni con le principali istituzioni internazionali: UN, UNICEF, WB.

IPEC e ILO promuovono quindi la prima pubblicazione statistica basata su stime dettagliate ed ufficiali circa il fenomeno del lavoro minorile, ottenute tramite la metodologia SIMPOC. Lo studio viene valutato da una commissione indipendente incaricata da UN: il riscontro è molto positivo, in particolare, si è evidenziata la proficua impostazione strategica della metodologia SIMPOC, in materia di collaborazione sinergica e sistematica con tutti i diversi istituti statistici nazionali.

(iii) 2004 - 2005: consolidamento della metodologia statistica

SIMPOC continua ad espandere ulteriormente il proprio orizzonte di indagine e di training nei confronti degli istituti statistici nazionali.

Entro la fine del 2005, SIMPOC redige più di 280 studi: questi includono 60 studi nazionali generalizzati riguardanti il lavoro minorile (National Household Survey - NHS), 110 studi valutativi riguardanti comparti economici specifici (Baseline Survey - BLS), 82 indagini preliminare di tipologia RA (Rapid Assessment Methodology ILO/UNICEF).

Nel corso di questa fase, si registrano molti segnali incoraggianti riguardo lo spessore della metodologia statistica sviluppata ed implementata presso i diversi studi condotti. Inoltre, un numero crescente di istituti statistici nazionali, implementano in modo sistematico i questionari riguardanti il lavoro minorile, presso le loro tradizionali e regolari attività di raccolta dei dati statistici a carattere socio-economico.

(iv) 2006-2008: definizione protocollo statistico standardizzato

Durante la " $17^{\text {th }}$ International Conference of Labour Statisticians" (ICLS) Ginevra 2003, ILO detta disposizioni riguardo lo sviluppo di un report a carattere scientifico-accadenico, inerente lo stato dell'arte riguardo le metodologie statistiche 
dedite alla rilevazione del lavoro minorile. Questo report ${ }^{16}$, verrà discusso in occasione della prossima " $18^{\text {th }}$ ICLS" che si terrà nel novembre-dicembre $2008{ }^{17}$.

L'obiettivo è quello di definire una serie di norme rigorose a livello internazionale, aventi l'obiettivo di rendere esattamente confrontabili ed omogenei, sia i dati che le misure, riguardanti il fenomeno del lavoro minorile: la qualità del metodo statistico, non a caso, è stata esattamente evidenziata quale elemento preliminare al fine di poter deliberare le migliori azioni di policy, tese al contrasto di tale fenomeno in tutti i paesi del mondo.

A questo stretto proposito, il progetto SIMPOC, si prefigge tre specifici obiettivi futuri:

i) Espandere le proprie valutazioni statistiche anche a variabili e dati di natura non prettamente economica (es. livello culturale, influenze religiose, prospettive storiografiche etc...), al fine di determinare in che misura queste particolari variabili concorrono nel determinare il fenomeno del lavoro minorile.

ii) Rivedere e affinare alcuni dei degli strumenti implementati presso la metodologia SIMPOC (es. modalità di intervista ai bambini) e sviluppare metodologie atte a consentire la stima attendibile riguardante le forme peggiori di lavoro minorile a livello nazionale.

iii) Definizione di indicatori appropriati, al fine di identificare il diverso grado di pericolosità implicato dal lavoro minorile.

Per quanto concerne la situazione finanziaria ${ }^{18}$ relativa ai fondi necessari per sviluppare e ed applicare la metodologia SIMPOC, ad oggi la situazione si dimostra in pieno equilibrio. Dal suo avvio nel 1998, il programma IPEC è stato in grado di attrarre un flusso costante di fondi destinati alla ricerca e finalizzatasi presso la metodologia SIMPOC. Gli introiti di cui attualmente può beneficiare SIMPOC, si basano su una rosa relativamente ristretta finanziatori istituzionali; in particolare, i finanziamenti provenienti dall'amministrazione USA (attraverso "US Department of Labor") costituiscono circa il $75 \%$ del budget totale a disposizione del programma.

\footnotetext{
${ }^{16}$ Lavori preparatori in ILO (2008).

$1718 \wedge$ ICLS web-page:

$<$ http://www.ilo.org/global/What_we_do/Statistics/events/icls/lang--en/docName--WCMS_092024/index.htm $>$.

${ }^{18}$ ILO (2006a).
} 
Figura 3: indagine SIMPOC per tipologia di regione

\begin{tabular}{|l|l|l|l|l|l|} 
Region & NHS & BLS & RA & Other & Total \\
\hline Asia and Pacific & 15 & 33 & 19 & 18 & 85 \\
\hline Developed Economies & 5 & 2 & 1 & 2 & 10 \\
\hline Latin America and Caribbean & 16 & 48 & 42 & 2 & 108 \\
\hline Middle East and North Africa & 1 & 2 & 1 & 1 & 5 \\
\hline Sub-Saharan Africa & 19 & 22 & 12 & 11 & 64 \\
\hline Transition Economies & 4 & 3 & 7 & -- & 14 \\
\hline Total & 60 & 110 & 82 & 34 & 286 \\
\hline
\end{tabular}

*NHS: National household survey; BLS: Baseline survey; RA: Rapid assessment

Figura 4: indagine SIMPOC per tipologia di regione

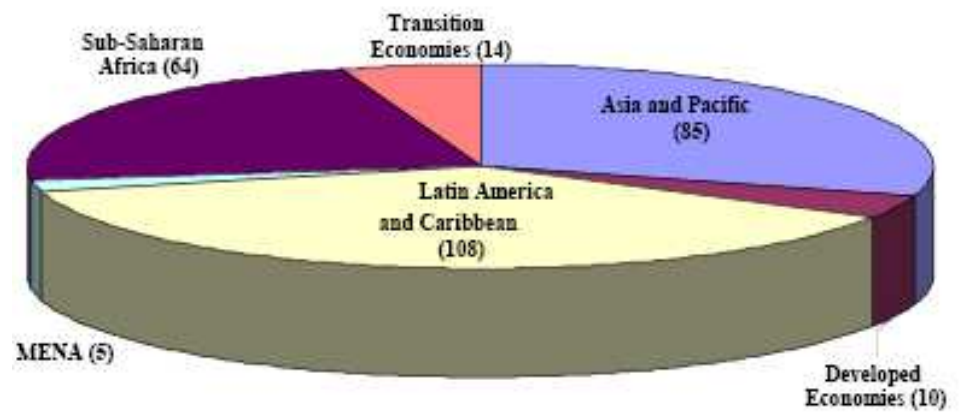

Figura 5: copertura globale indagini SIMPOC

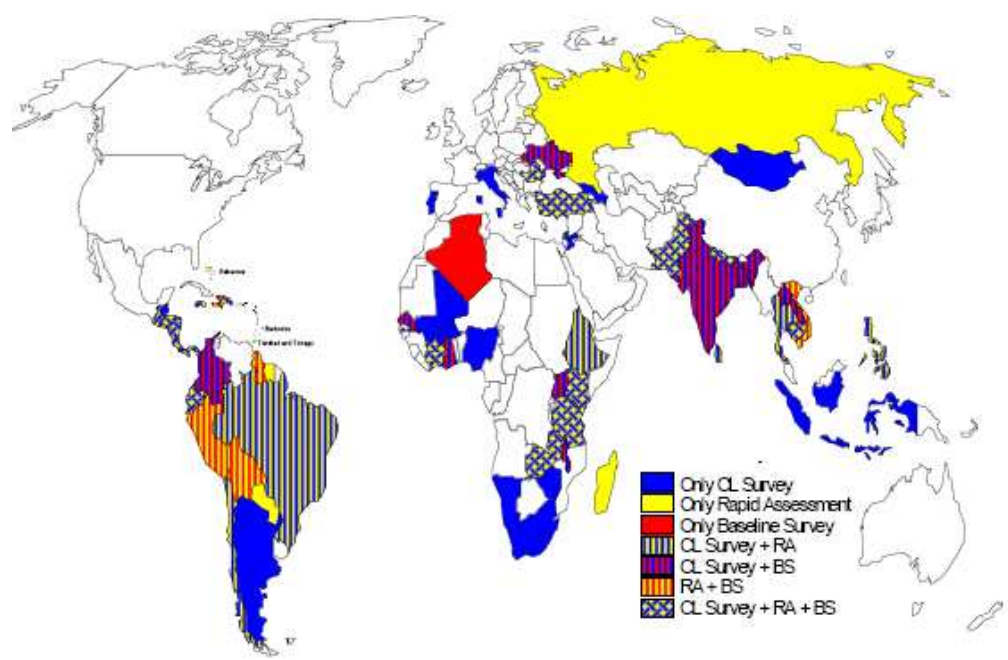




\section{World Bank - LSMS}

\section{"Living Standard Measurement Survey"}

Un seconda metodologia tesa a monitorare le dinamiche di lavoro minorile è stata proposta da World Bank (WB) a partire dal $1980^{19}$.

L'obiettivo principale della metodologia applicata dalla World Bank (WB) tramite l'indagine statistica (a vocazione 'multiscopo') Living Standard Measurement Survey $(\mathrm{LSMS})^{20}$, consta in particolar modo, nello stimare il rischio - a cui sono esposti i minori dell' area geografica oggetto d'analisi - circa la probabilità di cadere nello stato di lavoro minorile sul medio periodo.

Questa tipologia di indagine, che si incardina all'interno di un processo di rilevazione 'multiscopo', si basa sulla somministrazione di tre distinte tipologie di questionario.

Il primo questionario, focalizzato sulle caratteristiche della famiglia, raccoglie informazioni dettagliate riguardanti i membri del nucleo familiare in ottica micro.

Il secondo questionario è caratterizzato da un orizzonte di riferimento macro, ed è sottoposto ai rappresentati delle differenti comunità locali.

La terza tipologia di questionario è dedicata alla rilevazione dei dati salienti riguardanti le attività produttive e conseguentemente viene somministrato ai responsabili delle attività produttive.

Queste tre tipologie di questionari, talvolta vengono integrate tramite l'introduzione di un quarto questionario impiegato presso le realtà scolastico/educative.

Uno dei principali punti di forza della metodologia LSMS, consta nella capacità di raccoglie dati ed informazioni sia a livello individuale (micro), sia a livello aggregato (macro), tale da fornire ai ricercatori un data-set molto ampio caratterizzato da una gamma di informazioni individuali, che posso essere successivamente incrociate e verificate tramite il confronto con il secondo data-set a vocazione più prettamente macro.

Gli sviluppi più recenti che hanno caratterizzato LSMS, mettono in risalto la grande validità ed utilità dei dati qualitativi, ottenuti tramite il lavoro di interazione con i focus-group locali.

Inoltre, sono state sporadicamente tentate (ma con scarsi riscontri) indagini qualitative volte a isolare variabili qualitative (es. background culturale) che

\footnotetext{
${ }^{19}$ Grosh, Muzon (1996).

${ }^{20}$ ILO, UNICEF, WB (2001), Scott, Steele, Temesgen (2005).
} 
potessero proporsi come "indicatori sentinella" circa il rischio - che caratterizza i minori stanziati presso un determinato comprensorio oggetto d'analisi - di cadere nella condizione di lavoro minorile.

Lo standard LSMS attuale, utilizza l'approccio ILO/SIMPOC atto a determinare se un soggetto è stato economicamente/produttivamente attivo nel corso degli ultimi dodici mesi. A livello internazionale, questo orizzonte temporale è normalmente impiegato nell'attività di mappatura della dinamica salariale, dell'occupazione e della disoccupazione specificatamente presso il comparto agricolo. Questa precisa scelta metodologica trova forte fondamento, dato il carattere prettamente agricolo che caratterizza le economie dei PVS.

La numerosità campionaria dell'approccio LSMS è relativamente piccola. Per gli studi di tenore nazionale il campione varia da un minimo di 1.500 famiglie, ad un massimo di 5.000 presso le nazioni più popolose.

$\mathrm{Si}$ tratta normalmente di un campione stratificato a due stadi, basato essenzialmente sul frazionamento mediante criterio geografico/amministrativo (ups) ${ }^{21}$; nella seconda fase si estrarranno - in modalità casuale - le famiglie caratterizzate dalla presenza di minori (uss) che entreranno a far parte effettivamente del campione. Al variare dello specifico scenario nazionale di riferimento, si prevede l'eventuale individuazione di opportune unità autorappresentative.

In considerazione del fatto che la metodologia LSMS è implementata principalmente presso i PVS - tipicamente caratterizzati da uno scarso livello tecnologico - la compilazione del questionario presso le famiglie, è affidata a rilevatori direttamente e personalmente impegnati sul campo (nella maggior parte dei casi, si esclude l'adozione di metodologia CATI e/o CAPI). Per quanto riguarda i questionari rivolti ai referenti amministrativi locali nonché ai responsabili delle attività produttive, generalmente viene adottata la metodologia CATI.

Un fattore che caratterizza in modo significativo la raccolta dati presso i nuclei famigliari, consta nella prassi di sottoporre il questionario ad ogni membro della famiglia (anche minori), a fronte delle comprovate nozioni alfabetiche elementari.

Il questionario riguardante il nucleo familiare, è composto da batterie di domande aventi lo scopo di raccogliere dati circa la struttura demografica delle

\footnotetext{
${ }^{21}$ Si applica il criterio PPS (Probability Proportional to Size), Fabbris (1989).
} 
famiglie, le condizioni di alloggio, l'istruzione, la sanità, l'occupazione, la propensione al risparmio.

La persona designata dai membri della famiglia, quale rappresentante del nucleo familiare $^{22}$ è chiamata a fornire le risposte riguardanti le questioni generali che interessano l'intero nucleo familiare. Nella maggior parte delle sezioni del questionario, ad ogni membro della famiglia è chiesto di rispondere a livello individuale; tuttavia i genitori sono autorizzati a rispondere per i bambini in età prescolare. In alcune particolari tipologie di indagine LSMS, tuttavia, il capo famiglia è l'unico membro intervistato, ma questa opzione costituisce l'eccezione e non la regola. Uno dei principi fondamentali della metodologia Living Standard Measurement Survey è esattamente quello di garantire ai soggetti intervistati di poter rispondere liberamente a livello individuale: non è mai raccomandato che il capo famiglia risponda in nome e per conto di tutto il nucleo.

Per quanto concerne le domande circa il lavoro svolto durante l'ultimo arco temporale, le informazioni raccolte riguardano tipicamente le ore lavorate, la tipologia di attività nonché l'eventuale trattamento fiscale/previdenziale.

Come già anticipato, analoghi questionari sono proposti anche ai responsabili delle attività produttive, insediate nelle aree individuate presso le unità di primo stadio (ups).

Le indagini LSMS, atte ad analizzare le dinamiche di lavoro minorile, implicano la necessità di poter elaborare un ampio spettro di dati ed informazioni riguardanti l'effettiva attività scolastica dei minori, inferendoli dai risultati emersi rispettivamente dai questionari sottoposti delle famiglie, agli amministratori locali e ai datori di lavoro.

Attraverso l'analisi delle inferenze ottenute, basate sulle osservazioni emerse tramite l'indagine campionaria effettuata con l'ausilio delle tre differenti tipologie di questionario, il modello LSMS si pone l'obiettivo di stimare il tasso di rischio - a cui sono esposti i bambini dell'area geografica in questione - circa la probabilità di cadere nello stato di lavoro minorile nel medio periodo.

Parallelamente, per quanto riguarda le forme peggiori di lavoro minorile, le indagini LSMS si caratterizzano per il fatto di essere basate su di un campione numericamente relativamente modesto, il che rende - anche in questo caso difficoltosa l'analisi di tali caratteristiche di spiccato tenore qualitativo.

\footnotetext{
${ }^{22}$ Non necessariamente il padre/marito.
} 
La metodologia LSMS si propone quindi come l'unico protocolla statistico attualmente disponibile, adatto ad analizzare e descrivere la correlazione fra tenore di vita delle famiglie di origine e relativo rischio dei figli di essere interessati dalla dinamica di lavoro minorile.

\section{ISTAT "BAMBINI E LAVORI”: I'esperienza italiana}

Il lavoro minorile costituisce una criticità della quale ci si occupa quasi esclusivamente con riferimento ai PVS. Effettivamente, nei PVS tale fenomeno è sensibilmente più diffuso che in occidente. Tuttavia, il lavoro minorile si propone quale tematica di indiscussa rilevanza ed urgenza: in ragione di questa considerazione, anche ISTAT conduce periodicamente indagini $^{23}$ statistiche atte a fotografare questo fenomeno presso la realtà italiana.

La più recente ricerca ${ }^{24}$ ISTAT circa il lavoro minorile ad oggi disponibile, condotta nel biennio 2000-2002, si inserisce esattamente all'interno nell'alveo statistico-metodologico inaugurato da ILO/IPEC e caratterizzato della metodologia di rilevazione SIMPOC.

Il lavoro minorile su cui si è concentra tale esplorazione statistica, è quello statisticamente meno agevole da osservare, ovvero quello relativo ai bambini al disotto del 15 anno di età.

Vengono presi in considerazione tutti i minori classificabili come economicamente/produttivamente attivi secondo i dettami di ILO, ovvero tutti i bambini che abbiano svolto almeno un'ora di lavoro nel periodo di riferimento.

Nel caso del lavoro minorile, ILO ricorre normalmente a due diversi periodi di riferimento temporale: (i) la settimana o (ii) l'anno, ove quest'ultimo viene utilizzato con l'obiettivo di cogliere le numerose occupazioni occasionali svolte in genere dai bambini. Per questo stesso motivo, ed a causa della dimensione piuttosto ridotta che caratterizza il fenomeno in Italia, il periodo di riferimento ritenuto idoneo da ISTAT è stato l'anno.

Rientrano, quindi, nel concetto lavoro minorile preso in esame, sia tutte le attività economico-produttive formali svolte dai bambini, sia tutte quelle occupazioni

\footnotetext{
${ }^{23}$ Con particolare riguardo al segmento del lavoro sommerso, Zavanella (1999).

${ }^{24}$ Buggeri, ISTAT (2002).
} 
estemporanee (orizzonte privato-famigliare) che si ritiene possano avere riflessi negativi sulla salute, l'educazione e il normale sviluppo del minore.

Estranee all'oggetto di indagine Italiana, sono tutte attività di natura illegale che a vario titolo possono coinvolgere anche i minori.

La scelta metodologica ed operativa adottata in questa indagine, è stata quindi quella di cogliere il fenomeno nella sua estensione massima, per arrivare solo successivamente a distinguere al suo interno le diverse componenti, in modo da fornire una base informativa più accurata per eventuali policy.

Ad oggi , tra i paesi UE, solo il Portogallo ${ }^{25}$ si è cimentato in una analisi di tale tipologia. L'attività svolta da ISTAT è senza alcun dubbio pionieristica per quanto riguarda i paesi sviluppati, tant'è che l'individuazione e la definizione della metodologia statistica della ricerca, ha costituito essa stessa un obiettivo esplicito del progetto italiano.

\subsection{L'organizzazione della ricerca}

Considerata la sensibilità del fenomeno in questione, unita alla assoluta carenza di una tradizione statistica consolidata presso i paesi occidentali, ISTAT ha deciso di ricorrere a metodi alternativi di rilevazione del lavoro minorile e di far quindi derivare la quantificazione del fenomeno, tramite l'utilizzo sistemico di molteplici fonti statistiche.

In via schematica, il processo di analisi statistica, può essere suddiviso in quattro macro-fasi:

i) Analisi della letteratura pregressa redatta dall' ILO/IPEC - SIMPOC.

ii) Analisi di fenomeni secondari e derivati, correlati al fenomeno di lavoro minorile (es. abbandono scolastico etc..).

iii) Realizzazione di indagini campionarie dirette sui minori.

iv) Analisi sistemica di tutte le fonti statistiche disponibili.

Ogni singola fase, incorpora degli obiettivi specifici.

La prima fase, mira alla definizione preliminare dei riferimenti statisticometodologici dai quali attingere e conformarsi, durante tutto l'iter di rilevazione ed elaborazione dei dati.

\footnotetext{
${ }^{25}$ Analizzato in Invernizzi (2005).
} 
La seconda, di natura più spiccatamente qualitativa, ha consentito di ottenere una prima stilizzazione del fenomeno, con particolare riferimento alla fascia anagrafica più giovane che rientra nella coorte 6-10 anni. La ricerca infatti tiene conto anche di questa realtà, seppur senza avere l'ambizione di arrivare ad una quantificazione rigorosa di questo segmento così estremo e difficile da cogliere.

L'obiettivo della terza fase è stato invece quello di raccogliere informazioni - sia pure di natura proxy - riguardante il segmento più nascosto ed inaccessibile del lavoro minorile, che rischiava di sfuggire a qualsiasi indagine nella quale $i$ minori fossero chiamati a rispondere direttamente ad un intervistatore. La terza fase, per la quale la collaborazione con ILO/IPEC e SIMPOC è stata particolarmente feconda, ha riguardato la formalizzazione dell'indagine di tipo campionario, prettamente rivolta ai minori.

L'ultima fase, prevede l'interpretazione integrata e sistemica delle diverse fonti statistiche a disposizione presso il sistema statistico italiano, al fine di pervenire ad una rappresentazione d'insieme del fenomeno, sfruttando i diversi risultati intermedi emersi all'interno del progetto.

\subsection{Valutazione delle diverse tipologie di indagine}

Seguendo le indicazioni metodologico/operative dettate dal protocollo SIMPOC, sono state testate tre differenti tecniche d'indagine, rispettivamente incentrate su (i) nuclei famigliari, le (ii) istituzioni scolastiche nonché la (iii) tradizionale Rilevazione sulle Forze di Lavoro (RCFL).

In primo luogo, è stato inserito un approfondimento circa la tematica del lavoro minorile, all'interno della tradizionale Indagine Multiscopo ${ }^{26}$ presso le famiglie, dedicata all'infanzia e all'adolescenza.

Successivamente si è ritenuto opportuno approntare un'indagine sperimentale condotta presso gli istituti scolastici atta a coprire la coorte 12-15 anni.

Come terza modalità di indagine, è stato realizzato un apposito formulario - dal titolo Le Prime Esperienze di Lavoro dei Giovani - che è stato somministrato contestualmente alla tradizionale Indagine sulle Forze di Lavoro ${ }^{27}$ (RCFL).

La prima indagine ha fornito un quadro preliminare delle caratteristiche del fenomeno in Italia, ed ha fatto emergere l'esigenza di riconsiderare alcuni degli

\footnotetext{
${ }^{26}$ Efficace rassegna in Schizzerotto et. al. (2001).

${ }^{27}$ ISTAT (2004).
} 
strumenti adottati quando si vogliono intervistare direttamente i minori in un'indagine su vasta scala.

La necessità di introdurre alcuni correttivi e/o varianti, deriva essenzialmente dall'alto grado di inattendibilità delle risposte fornite dai minori: da una parte, infatti, la loro percezione del concetto di lavoro, risulta ancora estremamente sommaria, inoltre, il desiderio di sentirsi "grandi", induce sovente a sopravvalutare il proprio contributo all'interno della normale gestione familiare. L'effetto di entrambi gli atteggiamenti determina quindi un significativo ed ampio numero di risposte distorsive, tese a sovrastimare una effettiva attività lavorativa da parte dei minori italiani.

Una seconda tipologia di bias informativo, deriva dalla contestuale presenza di bambini e genitori durante le interviste. Si tratta anch'essa di una compresenza altamente distorsiva che - al contrario di quanto osservato al punto precedente - rischia di portare ad una sottostima del lavoro minorile, soprattutto per la sua componente più grave ed accentuata.

Le indagini sugli studenti presso gli istituti scolastici, in via teorica mostrano il chiaro vantaggio di consentire di intervistare i bambini senza l'influsso distorsivo provocato dalla presenza dei genitori, inoltre offre la possibilità di sfruttare un campione molto più ampio e quindi significativo.

Tuttavia, le doverose norme a tutela dei minori, prevedono che i genitori debbano essere preventivamente informati dell'intervista che sarà condotta all'interno dell'orario scolastico, reintroducendo - almeno in parte - l'interferenza della famiglia che si cerca di evitare.

La tecnica di indagine che mostra il miglior rapporto efficienza-efficacia, si dimostra essere la tipologia proposta in accompagnamento ed integrazione della tradizionale Rilevazione sulle Forze di Lavoro (RCFL).

\subsection{Notazioni metodologiche}

In primis, occorre sottolineare la relativa "parzialità" dei risultati che vengono forniti. Parzialità dovuta tanto al fatto che il contatto diretto con le forme più accentuate di sfruttamento minorile, può rivelarsi difficile se non addirittura impossibile, con l'evidente rischio che questo sfugga a gran parte dell'indagine. 
Le rilevazioni ISTAT presso le famiglie, si basano inoltre sulle liste anagrafiche, con una conseguente sottorappresentazione della popolazione straniera, in particolare se irregolare.

Una seconda cautela riguarda, più in generale, l'utilizzo di indagini presso le famiglie per l'analisi del lavoro minorile: in questo tipo di rilevazioni, il lavoro minorile vero e proprio - intendendo per questo lo sfruttamento dei minori - è suscettibile di emergere con insufficiente nettezza.

La tecnica di affiancare la statistica circa il lavoro minorile all'Indagine sulle Forze di Lavoro (RCFL), per quanto risponda esattamente alle indicazioni SIMPOC, non è sembrata quindi totalmente confacente al contesto culturale di un paese sviluppato. Si è conseguentemente posto il tema di come declinare in modo opportuno le raccomandazioni internazionali SIMPOC e ILO/IPEC, in modo da evitare o ridurre il rischio di "occultamento" del fenomeno. Conseguentemente, ISTAT ha quindi predisposto una ulteriore rilevazione, tesa a studiare la sola coorte 15-18 anni, somministrando questionari di natura retrospettiva, inerenti (i) primo lavoro svolto nonché (ii) tutte le età durante le quali si sono maturate esperienze di lavoro.

Questo accorgimento ha prodotto notevoli vantaggi: non cogliendo i ragazzi in "flagranza di reato", si sono ridotti drasticamente i bias derivanti da omissioni che possono sorgere circa la percezione di un fenomeno illegale. 
«Ci siamo accorti dei bambini. Non per motivi emozionali o morali, ma per significative considerazioni economiche. Se si vuole che la povertà sia drasticamente ridotta o eliminata bisogna centrare l'attenzione sulla generazione futura [...]. I programmi destinati ai bambini non devono assolutamente avere nulla di umanitario né di caritatevole, ma essere considerati una componente fondamentale dello sviluppo, proprio come (e forse ancor di più di) costruire fabbriche, autostrade $o$ aeroporti».

(M. Yunus, 2008 pp. 68-69) 


\section{BIBLIOGRAFIA}

Biggeri L., ISTAT (2002) (a cura di),

Bambini e Lavori, Sistema Informativo ISTAT, Roma.

Buffa F., Caracuta F. (2005),

Il lavoro Minorile: Problematiche Giuridiche, Halley Editrice, Macerata.

Fabbris L. (1989),

L'Indagine Campionaria, NIS, Roma.

Grosh M.E., Muzon J. (1996),

A Manual for Planning and Implementing the Lliving Standards Measurement Study Survey, LSMS Working Paper n.126, World Bank, Washington D.C.

ILO (2002),

Every Child Counts: New Global Estimates on Child Labour, ILO Publications Bureau, Ginevra.

ILO (2004a),

SIMPOC Manual for Child Labour Data Analysis and Reports, ILO Publications Bureau, Ginevra.

ILO (2004b),

Child Labour Statistics: Manual on Methodologies for Data Collection through Surveys, ILO Publications Bureau, Ginevra.

ILO (2006a),

SIMPOC Strategy Note 2006-2008, ILO Publications Bureau, Ginevra.

ILO (2006b),

SIMPOC Global Child Labour Trends 2000-2004, ILO Publications Bureau, Ginevra.

ILO (2007a),

IPEC Child Labour Statistics: The SIMPOC Programme, ILO Publications Bureau, Ginevra.

ILO (2008),

Child Labour Statistics, atti ILO Meeting of Experts on Labour Statistics, Ginevra, 1-10 April 2008.

ILO, UNICEF, WB (2001)(a cura di),

A Comparison of Survey Instruments for Collecting Data on Child Labour, UCW Project - CESIS Università di Roma "Tor Vergata", Roma.

Invernizzi A. (2005),

Perspectives on Children's Work in the Algarve (Portugal), Critical Social Policy, vol.25 (2005), pp.198-222. 
ISTAT (2004),

La Nuova Rilevazione sulle Forze di Lavoro, Sistema Informativo ISTAT, Roma.

Schizzerotto A., et. al. (2001)(a cura di),

Esperienza in Tema di Indagini Multiscopo ECHP, Rapporto di Ricerca Commissione per la Garanzia dell'Informazione Statistica, Roma.

Scott K., Steele D., Temesgen T. (2005),

LSMS Household Sample Surveys in Developing and Transition Countries, UN Statistics Division, New York.

Verma V. (forthcoming),

SIMPOC Sampling Manual: Sampling for Household-based Surveys of Child Labour, preliminary draft of ILO Manual on Sampling for Child Labour Surveys, Ginevra.

Yunus M. (2008),

Un mondo senza povertà, Feltrinelli, Milano.

Zavanella B. (1999),

Nuove possibilità di stima del lavoro sommerso attraverso l'uso congiunto di censimenti e registri, (mimeo) Convegno della Società Italiana di Statistica 1999, Udine.

\section{Allegati}

ILO (2007b),

Questionario SIMPOC-IPEC "Essential Questions on Child Labour for Household Surveys - up date version 17/07/2007”, ILO Publications Bureau, Ginevra. 